\title{
MERS epidemiological investigation to detect potential mode of transmission in the 178th MERS confirmed case in Pyeongtaek, Korea
}

\author{
Kyujin Chang ${ }^{1}$, Moran $\mathrm{Ki}^{2}$, Eun Gyu Lee ${ }^{3}$, Soon Young Lee ${ }^{4}$, Byoungin Yoo ${ }^{5}$, Jong Hyuk Choi ${ }^{6}$ \\ ${ }^{1}$ Independent Health Consultant, Suwon; '2Department of Cancer Control and Policy, Graduate School of Cancer Science and Policy, National \\ Cancer Center, Goyang; '3ivsion of Epidemiology and Health Index, Center for Genome Science, Korea Centers for Disease Control and \\ Prevention, Cheongju; ${ }^{4}$ Department of Preventive Medicine and Public Health, Ajou University School of Medicine, Suwon; ${ }^{5}$ Department of \\ Preventive Medicine, Soonchunhyang University College of Medicine, Cheonan; ${ }^{6}$ Department of Preventive Medicine, Dankook University \\ College of Medicine, Cheonan, Korea
}

\begin{abstract}
Most cases of Middle East Respiratory Syndrome (MERS) infection in Korea (outbreak: May 11-July 4, 2015) occurred in hospital settings, with uncertain transmission modes in some cases. We performed an in-depth investigation epidemiological survey on the 178th case to determine the precise mode of transmission. A 29year-old man living in Pyeongtaek presented on June 16 with a febrile sensation, chills, and myalgia. Upon confirmatory diagnosis on June 23, he was treated in an isolation room and discharged on July 2 after cure. An epidemiological investigation of all possible infection routes indicated two likely modes of transmission: exposure to MERS in Pyeongtaek St. Mary's Hospital during a visit to his hospitalized father (May 18-29), and infection through frequent contact with his father between the latter's referral to Pyeongtaek Good Samaritan Bagae Hospital for treatment without confirmatory diagnosis until his death (May 29-June 6). Although lack of clear proof or evidence to the contrary does not allow a definitive conclusion, all other possibilities could be excluded by epidemiological inferences. While it is impossible to trace back the modes of transmission of all cases in a large-scale outbreak, case-by-case tracking and isolation of infected individuals and those in close contact with them is important in preventing the spread. Efforts should be made to establish a methodology for rapid tracking of all possible contacts and elimination-based identification of the precise modes of transmission.
\end{abstract}

KEY WORDS: Middle East Respiratory Syndrome, Epidemiologic investigation, Mode of transmission, Outbreak, Korea

\section{INTRODUCTION}

Middle East Respiratory Syndrome (MERS) was first detected in South Korea (hereafter Korea) in May 2015, and resulted in a death toll of 36, with 186 confirmed cases between May 11

\section{Correspondence: Moran Ki}

Department of Cancer Control and Policy, Graduate School of Cancer

Science and Policy, National Cancer Center, 323 Ilsan-ro, Ilsandong-gu, Goyang 10408, Korea

Tel: +82-31-920-2736, Fax: +82-50-4069-4908, E-mail: moranki@naver.com

Received: Jul 30, 2015, Accepted: Aug 15, 2015, Published: Aug 15, 2015

This article is available from: http://e-epih.org/

(C) 2015, Korean Society of Epidemiology

(C) This is an open-access article distributed under the terms of the Creative Commons Attribution License (http://creativecommons.org/licenses/by/3.0/), which permits unrestricted use, distribution, and reproduction in any medium, provided the original work is properly cited. and July 4, the symptom onset dates of the first and last cases, respectively [1]. MERS is caused by a recombinant coronavirus strain (MERS-CoV) that was first identified in 2012; accumulating evidence points to dromedary camels as the likely major reservoir. While camel-to-human infection and subsequent human-to-human infection have been confirmed, the exact modes of transmission of MERS-CoV have yet to be clarified [2]. The origin of the outbreak in Korea was the arrival of the first case in May 2015 from Saudi Arabia, experiencing a high incidence of MERS. Most infections have been confirmed to occur in hospital settings [3]. However, some cases have unclear routes or symptom onset dates, necessitating an in-depth investigation. While it is difficult to clearly trace back all cases in a large-scale outbreak, an exclusion-based rapid investigation after mapping of all possibilities is indispensable to control outbreak.

The present study relates to an in-depth investigation perform- 
ed on the 178th case with confirmed MERS (hereafter referred to as \#178) who was infected during the 2015 outbreak in Korea. \#178 was followed starting from June 23, the date of confirmed diagnosis, and measures were taken to prevent further spread, with concerted efforts by an epidemiological investigation team of the Korea Centers for Disease Control and Prevention (KCDC) and civil professionals.

\section{MATERIALS AND METHODS}

This survey was performed using elimination methods after mapping all possible routes. As \#178 did not have direct contact with MERS patients, we investigated the possibilities for exposure from the 2-week incubation period (June 2-16) to the date of symptom onset. \#178 frequently visited his father during the latter's treatment in Pyeongtaek St. Mary's Hospital (PSMH) from May 18-29, and Pyeongtaek Good Samaritan Bagae Hospital (GSBH) from May 29 through June 6. We reviewed the investigation data of these hospitals and performed additional surveys on patients and related persons with whom \#178 may have come into contact. All persons with whom \#178 may have had contact during the 2 -week incubation period, e.g., his family, and colleagues, were asked in person or by phone whether they had MERS-related symptoms or visited a hospital for similar symptoms. Moreover, initial symptoms onset of \#178 were reexamined by checking his hospital visit and medical records. The KCDC performed polymerase chain reaction (PCR) assays on sputum samples and serum antibody detection tests on individuals with whom \#178 had direct contact, to screen for positive individuals with a confirmatory test.

Because this study was conducted as an outbreak investigation, institutional review board approval was not necessary.

\section{RESULTS}

\section{Clinical course}

\#178 is a 29-year-old man living in Pyeongtaek, Gyeonggido, Korea, where the initial outbreak occurred. He was working at a construction site in Asan, Chungcheongnam-do. He was a smoker and had social alcohol drinking, and was in good health without medication. He first noticed a febrile sensation, chills, and myalgia on June 16, and presented to Asan Saewoori Clinic on June 17, where he was prescribed medicine for an upper respiratory tract infection. On June 18, he was administered intravenous fluids for fever $\left(38.5^{\circ} \mathrm{C}\right)$ and myalgia in GSBH, again received outpatient treatment at Saewoori Clinic on June 19, and was again administered intravenous fluids on June 20 at GSBH. On June 21, owing to ongoing symptoms, he was ad- mitted to an isolation room in Pyeongtaek Good Morning Hospital, on referral from GSBH. Upon confirmatory diagnosis of MERS on June 23, he was then referred to Gyeonggi Provincial Medical Center Suwon Hospital.

The chest radiography taken on June 21 revealed that pneumonia had developed over a few days, and he was treated primarily for pneumonia. With negative results from two sputum tests and complete disappearance of symptoms, he was discharged on July 2 (Figure 1 ).

\section{Suspected exposure events and transmission routes \#178's father}

\#178's father (a resident of Jeollanam-do) was receiving postoperative pain management and chemotherapy. He had a history of liver cancer operation in Chonnam National University Hospital in 2008, and hepatic artery embolization for recurrent liver cancer and lung metastasis in Seoul Asan Medical Center in June 2014. He was hospitalized in PSMH from May 18-29, 2015 because of poor general condition. He was referred to GSBH on May 29 and died there on June 6. He was tested twice for MERS-CoV (sputum on May 29 and endotracheal aspiration on June 1) before his death, with negative results, and chest radiography (May 29) did not yield clear pneumonia findings. However, he developed fever on June 2 and the chest radiography taken on June 4 showed findings suspicious pneumonia. And no additional MERS test was performed after then. He was completely bed-ridden throughout hospitalization. He was admitted to Room 8104 in PSMH the day after discharge of \#1 from the same room. For the next 2 days, he shared Room 8112 with the \#9. There was no MERS patient in Room 7110, where he stayed for the next 8 days (Tables 1 and 2, Figures 2 and 3).

\section{Pyeongtaek St. Mary's Hospital}

During the time when \#178 visited his father in PSMH (May 18 -29), he might be exposed to MERS patients (\#6, \#9, \#11,

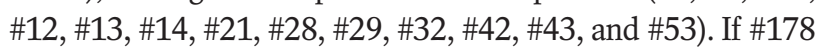
was infected in PSMH by May 29, the incubation period had exceeded 2 weeks due to showing the symptoms on Jun 16 (Tables 1 and 2, Figures 2 and 3).

\section{Pyeongtaek Good Samaritan Bagae Hospital}

The possibility that \#178 was infected by other MERS patients in GSBH during the period he visited his father (May 29June 6) was investigated. Three cases (\#22, \#52, and \#119) were hospitalized or visited emergency room during this time. Considering \#178's visiting times and movements with closed-circuit television data, \#52 and \#119 could be safely excluded. \#22 stayed for 14 hours in the room just opposite the room of \#178's father. However, \#22 did not leave the room, and there was no contact between \#22 and \#178 (Tables 1 and 2, Figure 2). 


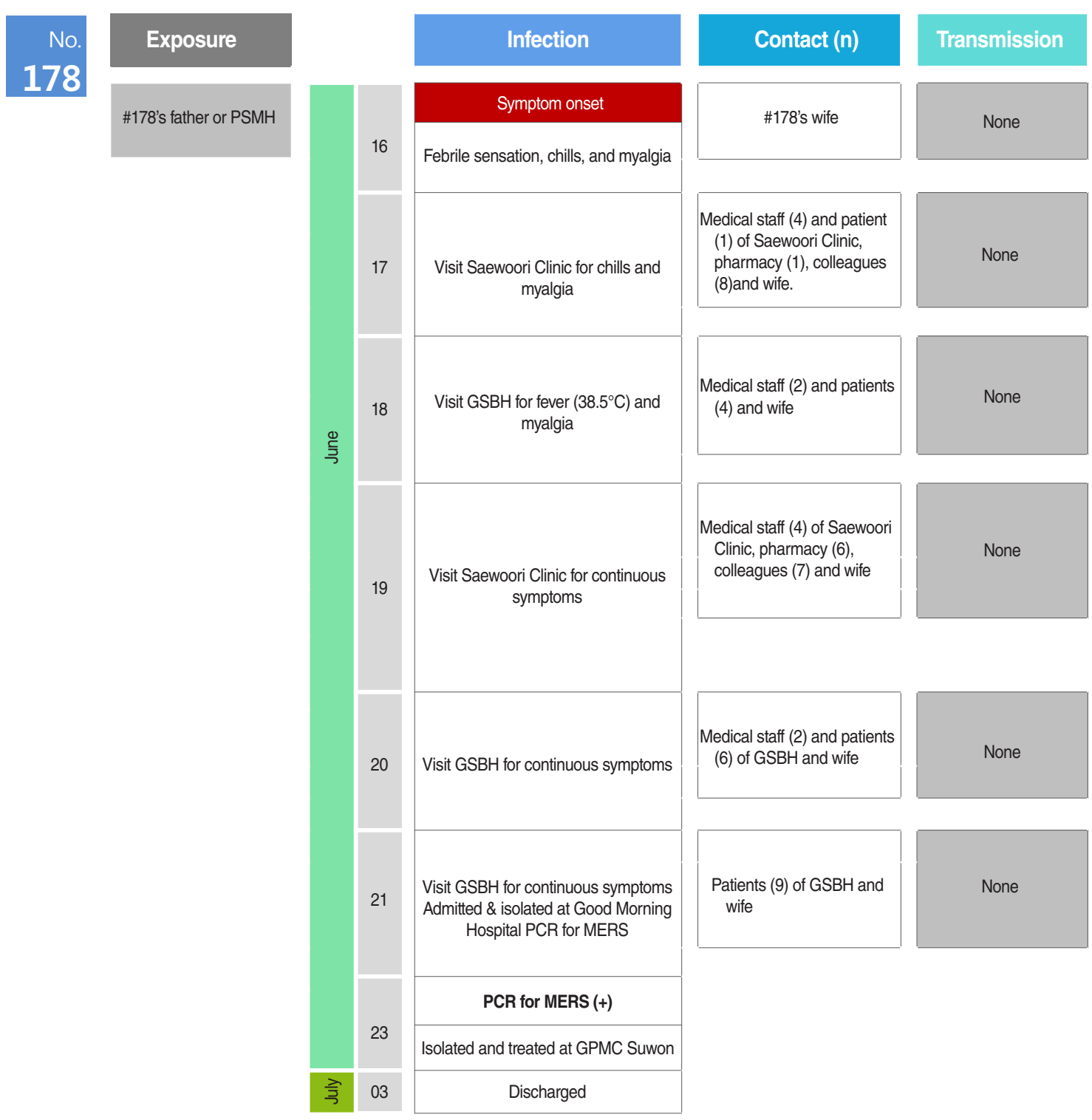

Figure 1. Main events by date for the 178th MERS case in Korea. MERS, Middle East Respiratory Syndrome; PSMH, Pyeongtaek St. Mary's Hospital; GSBH, Good Samaritan Bagae Hospital; PCR, polymerase chain reaction; GPMC Suwon, Gyeonggi Provincial Medical Center Suwon Hospital.

\section{\#178's wife and mother}

Because \#178's wife and mother provided care for \#178's father during hospitalization, we evaluated the possibility that they became infected and transmitted MERS-CoV to \#178. In particular, \#178's wife received outpatient treatment for upper respiratory symptoms 4 times (May 25, 27, 30, and June 3), which raised the suspicion that she was a mild case of MERS [4]. However, four sputum samples (June 26-July 2) from her yielded negative results, as did the serum test (June 25). And \#178's mother had no symptoms and showed negative sputum result, without no serum test (Tables 1 and 2, Figure 2).

\section{Pyeongtaek community}

During the incubation period for \#178, many MERS patients were in Pyeongtaek. Therefore, the possibility of infection in community was evaluated. We investigated the movements of 4 cases (\#32, \#40, \#53, and \#119) who were possibly active in the downtown of the same district around June 2-6. We considered the time of symptom onset (June 16) in \#178, and compared this with the times of possible exposure. The period between June 6 (afternoon) and June 15 was excluded because \#178 was in Jeollanam-do for his father's funeral. The comparison of movements yielded the following results: while there 
Table 1. The possible modes of transmission by exposure for the 178th MERS case

\begin{tabular}{|c|c|c|c|c|c|}
\hline No. & Exposure & Mode of transmission & Supporting evidence & Refuting evidence & Conclusion \\
\hline 1 & \#178's father & $\begin{array}{l}\text { \#178's father infected in PSMH } \\
(5 / 18-5 / 29) \rightarrow \text { father to \#178 in } \\
\text { GSBH (5/29-6/6) }\end{array}$ & $\begin{array}{l}\text { Incubation period, chest } \\
\text { radiography (r/o pneumonia), } \\
\text { mild fever }\end{array}$ & $\begin{array}{l}\text { Vague results on chest radiography, } \\
\text { only mild fever, } 2 \text { times PCR (-) of } \\
\text { father, all PCR (-) of other family } \\
\text { members }\end{array}$ & $\begin{array}{l}\text { Possible but unknown } \\
\text { due to limitation of } \\
\text { serum test }\end{array}$ \\
\hline 2 & $\mathrm{PSMH}$ & $\begin{array}{l}\text { \#178 infected in PSMH with long } \\
\text { incubation period }\end{array}$ & Statistically possible & Only suggestion & Possible \\
\hline \multirow[t]{2}{*}{3} & GSBH & $\begin{array}{l}\# 178 \text { infected in GSBH from other } \\
\text { cases (\#52, \#119) }\end{array}$ & Within incubation period & $\begin{array}{l}\text { Staying in hospital in different time } \\
\text { and different room }\end{array}$ & Unlikely \\
\hline & & $\begin{array}{l}\text { \#178 infected in GSBH from other } \\
\text { case (\#22) }\end{array}$ & $\begin{array}{l}\text { Incubation periods, rooms } \\
\text { of father and \#22 are near }\end{array}$ & $\begin{array}{l}\text { No contact between \#178 and \#22 } \\
\text { on CCTV }\end{array}$ & Unlikely \\
\hline 4 & \#178's wife & $\begin{array}{l}\text { \#178's wife infected in PSMH } \\
\text { (5/18-5/29) and transmitted to \#178 }\end{array}$ & $\begin{array}{l}\text { Her upper respiratory symptoms } \\
\text { (5/25-6/3), incubation period, } \\
\text { order }\end{array}$ & $\begin{array}{l}\text { MERS PCR (-) } 4 \text { times, but late } \\
\text { exam (6/26-7/2), MERS serology } \\
(-)\end{array}$ & Unlikely \\
\hline 5 & \#178's mother & $\begin{array}{l}\text { \#178's mother infected in PSMH } \\
\text { (5/18-5/29) and transmitted to \#178 }\end{array}$ & Within incubation period & $\begin{array}{l}\text { MERS PCR (-) and no upper respi- } \\
\text { ratory symptoms }\end{array}$ & Unlikely \\
\hline 6 & $\begin{array}{l}\text { Pyeongtaek } \\
\text { community }\end{array}$ & $\begin{array}{l}\text { Community acquired infection of } \\
\text { \#178 }\end{array}$ & $\begin{array}{l}\text { Four MERS cases were in } \\
\text { Pyeongtaek at that period. }\end{array}$ & $\begin{array}{l}\text { No evidence of staying in same } \\
\text { area of community }\end{array}$ & Unlikely \\
\hline
\end{tabular}

MERS, Middle East Respiratory Syndrome; PSMH, Pyeongtaek St. Mary's Hospital; GSBH, Good Samaritan Bagae Hospital; r/o, rule out; PCR, polymerase chain reaction; CCTV, closed-circuit television.

Table 2. Flow chart of the 178th MERS case and his family members

\begin{tabular}{|c|c|c|c|c|}
\hline Date & & \#178 & \#178's father (liver cancer patient) & \#178's wife \\
\hline \multirow[t]{12}{*}{ May } & 18 & Visit father/Room 8104 & Admission at Room 8104 of $\mathrm{PSMH}^{1}$ & \multirow{3}{*}{ Takes care of father-in-law/Room 8104 of PSMH } \\
\hline & 19 & Visit father & Room 8104 of PSMH & \\
\hline & 20 & Visit father & Move to Room 8112 & \\
\hline & 21 & Visit father/Room 8112 & Room 8112 & Nursing/Room 8112 \\
\hline & $22-24$ & Visit father/Room 7110 & Move to Room 7110 & \\
\hline & 25 & Visit father & Room 7110 & $\mathrm{R} / \mathrm{Sx} \mathrm{OPD}$ \\
\hline & 26 & Visit father & Room 7110 & \multirow{4}{*}{$\begin{array}{l}\text { Takes care of father-in- } \\
\text { law/Room } 7110\end{array}$} \\
\hline & 27 & Visit father & Room 7110 & \\
\hline & 28 & Visit father & Room 7110 & \\
\hline & 29 & Visit father/GSBH & Transfer to GSBH & \\
\hline & 30 & Visit father & MERS PCR (-)/GSBH & \multirow{3}{*}{$\begin{array}{l}\text { Takes care of father-in- } \\
\text { law/GSBH }\end{array}$} \\
\hline & 31 & Visit father & GSBH & \\
\hline \multirow[t]{9}{*}{ June } & 1 & Visit father & MERS PCR (-)/GSBH & \\
\hline & 2 & Visit father & GSBH & \multirow{4}{*}{ Takes care of father-in-law/GSBH } \\
\hline & 3 & Visit father & CXR pneumonia, vague result/GSBH & \\
\hline & 4 & Visit father & GSBH & \\
\hline & 5 & Visit father & GSBH & \\
\hline & 6 & Visit father & Death & Nursing/GSBH \\
\hline & $7-14$ & Residence in Jeollanam-do for funeral & & Residence in Jeollanam-do for funeral \\
\hline & 15 & Return to Pyeongtaek & & Return to Pyeongtaek \\
\hline & 16 & MERS symptom onset & & \\
\hline
\end{tabular}

MERS, Middle East Respiratory Syndrome; PSMH, Pyeongtaek St. Mary's Hospital; R/Sx OPD, respiratory symptom, positive, outpatient department; GSBH, Good Samaritan Bagae Hospital; PCR (-): polymerase chain reaction, negative; CXR, chest radiography.

${ }^{1}$ Patient zero (\#1) was in Room 8104, May 15-17.

was no overlapping area with \#40, contacts with \#32 (June 2), \#53 (June 2 and 3), and \#119 (June 2 and 4) could not be excluded because they were not isolated, and movement analyses could not clearly prove that they had no overlapping zones (Tables 1 and 2, Figure 2).
Preventive measures for those in contact with \#178

Eighty-one individuals who had contact with \#178 after symptom onset, including his wife, colleagues, and medical staffs, were quarantined. No additional MERS transmission was observed through the maximum possible incubation period (Figure 1). 


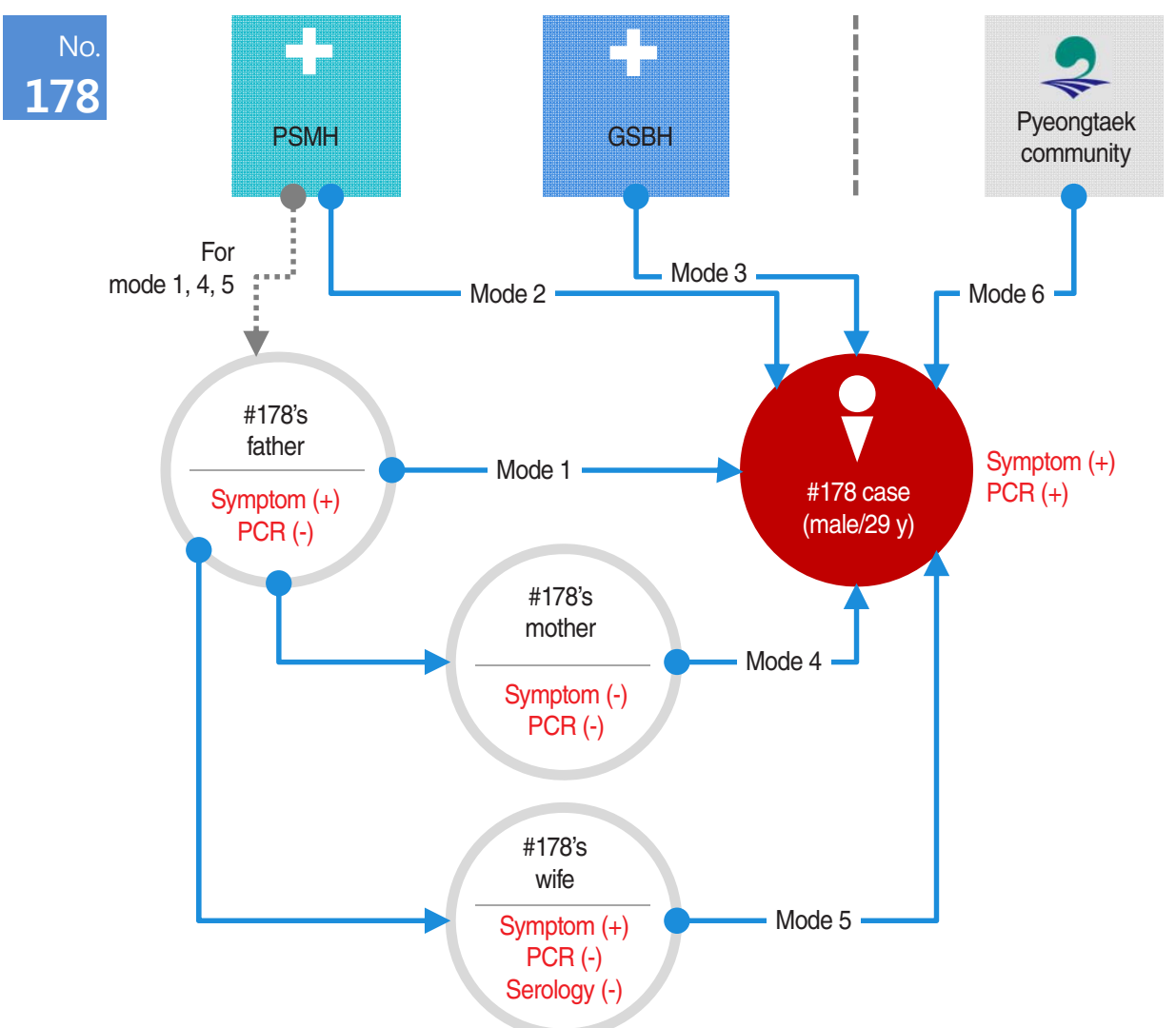

Figure 2. Possible modes of transmission of the 178th MERS case (see Table 2 for the number of transmission mode). MERS, Middle East Respiratory Syndrome; PSMH, Pyeongtaek St. Mary's Hospital; GSBH, Good Samaritan Bagae Hospital; PCR, polymerase chain reaction.

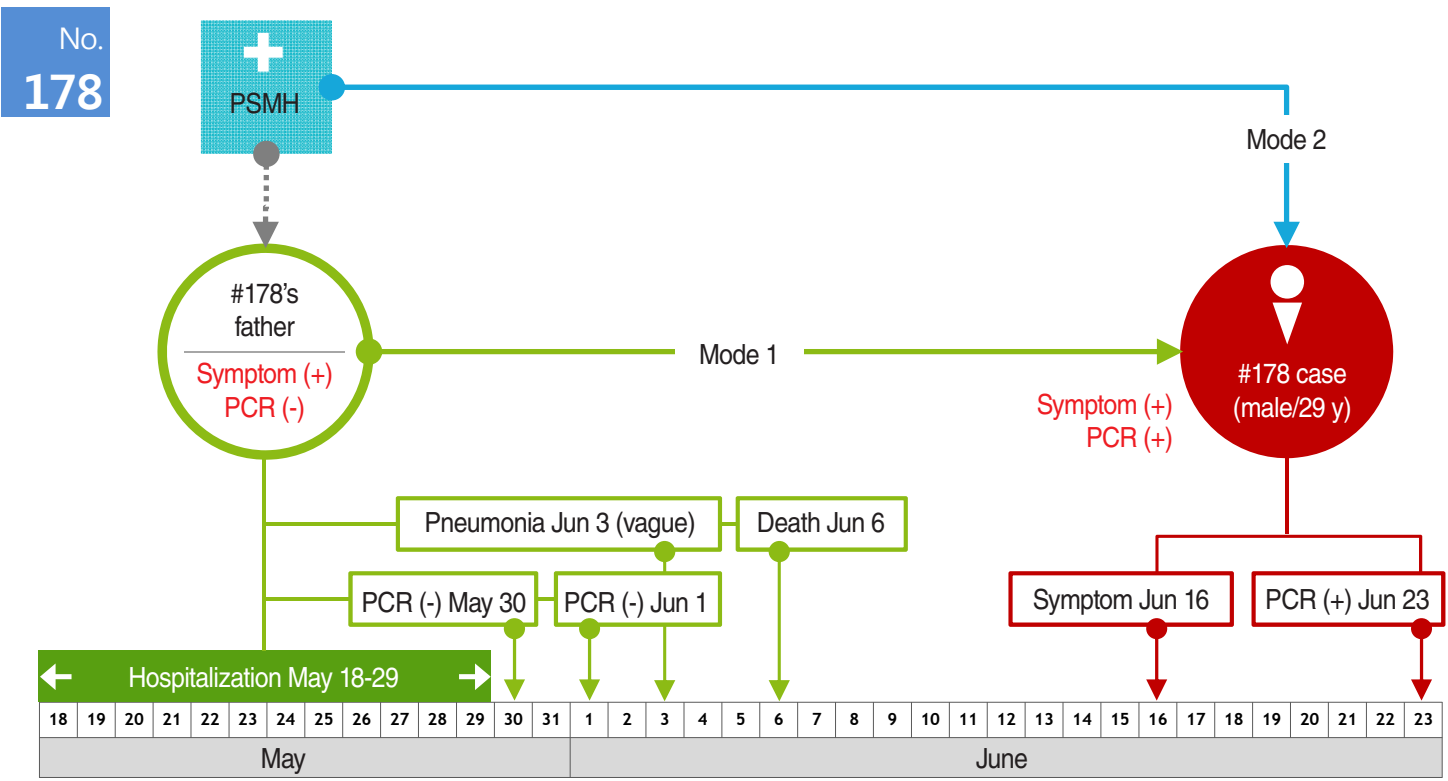

Figure 3. Two probable modes of transmission of 178th MERS case (see Table 2 for the number of transmission mode). MERS, Middle East Respiratory Syndrome; PSMH, Pyeongtaek St. Mary's Hospital; PCR, polymerase chain reaction. 


\section{DISCUSSION}

We performed an in-depth investigation on \#178 who had unclear modes of transmission. The results yielded two possibilities: infection during visits to his father who was a suspected false negative patient, and infection from other MERS patients in the hospital under the assumption that the incubation period was exceptionally long (Figure 3).

If it is postulated that \#178 was infected through his father, three scenarios may be considered for his father's infection: i) from the contaminated environment during the 2 days in Room 8104; ii) from the other patients during the 2 days in Room 8112; and iii) from repeated exposure to MERS-CoV in other areas within the hospital. If his father was actually a false negative patient, a plausible scenario would be that \#178 was infected from his father during visits, especially around June 6, the date of his father's death. The scenario is plausible in terms of an incubation period of 2-14 days [2]. However, except for vague findings of pneumonia, his father showed no clear MERS symptoms, and the sputum test was negative, as were those of 5 other family members contacted his father. As his father is dead, it could not be verified whether he was a patient. On a related note, there was a comparable case in Tunisia in 2013. A 66-yearold man developed high fever on returning home after meeting his 30-year-old daughter in Qatar, and died 5 days after hospitalization (12 days after symptom onset). The MERS PCR test performed the day before his death was negative. His 34-yearold son took care of him during his illness, developed MERS symptoms with high fever 3 days after the funeral, and was confirmed. The son had no foreign travel, and as this was the first MERS case in Tunisia, the only possibility was infection from the father. The Tunisian public health office sent the father's serum taken before his death to the US Centers for Disease Control, and it was MERS-positive [4]. In Korea, there was also a \#119 case in which a negative diagnosis on the first test was corrected to positive with additional tests. This suggests that it is possible \#178 was infected from his father. Unlike the Tunisian case, a confirmatory test was not possible because the father's serum was not stored [5].

The second scenario assumes a long incubation period. The estimated incubation period in this scenario is 18 days, which is considerably longer than the known 2-14 days, but is statistically possible.

Other modes of transmission were evaluated and excluded. First, the possibility of being infected from the other 3 MERS patients in GSBH could be excluded by confirming no direct contact. The possibility of \#178's wife or mother infecting him after being infected from his father could be excluded, considering the investigation results. Community-acquired infection is possible, but it is less plausible than others, more so considering the MERS-specific transmission patterns are in hospital settings and through close contact [6]. The possibility of error regarding \#178's symptom onset date was also considered; however, we could exclude this revealing that he did not have any symptoms before June 16 , and that he did not visit medical facilities before June 16 (Table 2).

From the standpoint of prevention, there are lessons learned. Although \#178 and his family were exposed to MERS-CoV in $\mathrm{PSMH}$, and belonged to the subjects for initial quarantine, they were not closely monitored. Although she received outpatient treatment for respiratory symptoms several times, \#178's wife was not subjected to MERS testing. His family kept providing care for \#178's father in GSBH without precautionary measures, even after transfer from PSMH. No additional MERS testing was undertaken after the sputum-negative for \#178's father.The funeral was held without any precautionary measures. After \#178 returned to Pyeongtaek following the funeral, he came into contact with a large number of individuals after symptom onset. Fortunately, no additional MERS cases occurred among those in contact with \#178. In addition, those in contact with \#178's father, including relatives, GSBH staffs, and those who transported or shrouded the corpse, did not develop any symptoms.

In conclusion, we arrived at two plausible scenarios: intrafamilial spread through the father and a prolonged incubation period from the infection at PSMH.

\section{ACKNOWLEDGEMENTS}

We owe a great debt of gratitude to the Pyeongtaek Community Health Center (Director: Byung-Seong Jeong) and responsible persons in each hospital for their committed cooperation in implementing this in-depth epidemiological investigation. Our sincere thanks also go to Eui-Jun Lee MD/EIS and YoungWoo Ryoo MD/EIS of the Gyeonggi-do Government for the approval to use the initial data of this paper and for his kind support with his expertise in epidemiology. Above all, we would like to express our sincere sympathies and condolences to the patient who went through treatment after the confirmatory diagnosis of MERS and his family for their sufferings and the loss of his father.

\section{CONFLICT OF INTEREST}

The authors have no conflicts of interest to declare for this study. 


\section{SUPPLEMENTARY MATERIAL}

Supplementary material (Korean version) is available at http: //www.e-epih.org/.

\section{REFERENCES}

1. Moran Ki. 2015 MERS outbreak in Korea: hospital-to-hospital transmission. Epidemiol Health 2015;37:e2015033.

2. Zumla A, Hui DS, Perman S. Middle East respiratory syndrome. 2015. doi: 10.1016/S0140-6736(15)60454-8.
3. Choi JW, Kim KH, Cho YM, Kim SH. Current epidemiological situation of Middle East respiratory syndrome coronavirus clusters and implications for public health response in South Korea. J Korean Med Assoc 2015;58:487-497 (Korean) .

4. Memish ZA, Al-Tawfiq JA. Middle East respiratory syndrome coronavirus infection control: the missing piece? Am J Infect Control 2014; 42:1258-1260.

5. Abroug F, Slim A, Ouanes-Besbes L, Hadj Kacem MA, Dachraoui F, Ouanes I, et al. Family cluster of Middle East respiratory syndrome coronavirus infections, Tunisia, 2013. Emerg Infect Dis 2014;20:15271530.

6. Al-Tawfiq JA, Memish ZA. Middle East respiratory syndrome coronavirus: epidemiology and disease control measures. Infect Drug Resist 2014;7:281-287. 